\title{
TREATMENT OF THE PERIODICALLY INHOMOGENEOUS SURFACE: MULTIPLE BOUNDARY CONDITION
}

\author{
H. Puszkarski* and M. Krawczyk \\ Surface Physics Division, Faculty of Physics, Adam Mickiewicz University \\ Umultowska 85, 61-614 Poznań, Poland \\ (Received March 14, 2000)
}

\begin{abstract}
We deal with the following question: how can the composite nature of a boundary condition formulated for a periodically inhomogeneous surface and involving the composite surface parameter, be treated analytically? We show that when the appropriate Fourier transformation is applied, the composite boundary condition reduces to a specific eigenproblem condition, which constitutes the spectrum of eigenvalues of an "effective" surface parameter, a novel quantity we introduced to account for the nonhomogeneity of the surface.
\end{abstract}

PACS numbers: $68.35 .-\mathrm{p}, 02.60 . \mathrm{Lj}$

\section{Introduction}

Recent years witnessed considerable interest in the study of properties of composite materials like photonic [1-3], phononic [4-8] or magnonic [9-11] crystals. Some of these materials consist of periodic lattice composed of identical parallel rods disposed in a homogeneous medium. If such structures are limited by planes perpendicular to the axis of the rods, inhomogeneous surfaces are created; the inhomogeneity of the surfaces in question has a periodical topology, since the cross-sections of the rods form a two-dimensional lattice. In such truncated (two-dimensional) periodic media (see e.g. [12]) the proper treatment of the inhomogeneous surface conditions becomes essential.

The problem resembles in some sense those problems, which have already been treated numerically in the literature, namely, we have in mind the problem of acoustic waves propagating at the periodically corrugated interface [13] or those associated with the surface of a substrate supporting a periodic array of wires [14]. To our best knowledge, there has been no successful attempt in the literature up to now, of finding an analytical way to solve the problem of inhomogeneous surface conditions. In this paper we propose an original treatment of periodically inhomogeneous surface conditions, by having recourse to the Fourier transformations.

*corresponding author; e-mail: henpusz@amu.edu.pl 


\section{The periodically inhomogeneous surface}

We consider the semi-space bounded by a flat surface $(x, y)$ with the $z$-axis perpendicular thereto. We express the radius vector in the form $r=\left[r_{\|}, z\right]$, where the two-dimensional vector $r_{\|}$defines the position of a point in the plane parallel to the surface, and let the coordinate $z_{0}$ determine the disposition of the surface limiting the semi-space. Let us also assume the physical medium filling the semi-space as homogeneous (with regard to some property) to the exception of the surface which we assume as periodically inhomogeneous. We shall postulate that the function $U\left(r_{\|} ; z\right)$ describing the physical process of interest shall fulfill the following boundary condition on the surface:

$$
\frac{\partial U\left(r_{\|} ; z\right)}{\partial z}+a\left(r_{\|} ; z\right) U\left(r_{\|} ; z\right)=0 \text { for } z \equiv z_{0} .
$$

Equation (1), as the equation which has to be fulfilled on the surface plane $z \equiv z_{0}$, is a boundary condition (of the Sturm-Liouville type) with a boundary parameter $a\left(r_{\|}\right)$being the following function of position:

$$
a\left(r_{\|}\right)=a_{I} S\left(r_{\|}\right)+a_{M}\left[1-S\left(r_{\|}\right)\right] .
$$

Thus we assume the surface to present a two-component composite structure (see Fig. 1) consisting of the space $M$ to which we shall refer as the background ("matrix") and spaces $I$ periodically disposed thereon ("impurity"). The function $S\left(r_{\|}\right)$

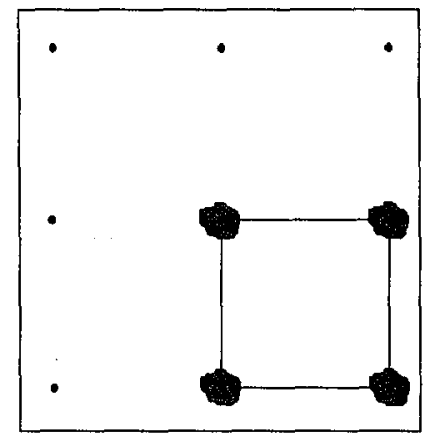

Fig. 1. A two-component composite surface structure; grey areas are periodically distributed, forming a square two-dimensional lattice (black points).

occurring in Eq. (2) takes the value 1 inside $I$ and 0 elsewhere. Thus the surface parameter is a periodic jump function taking only two values, namely $a_{I}$ and $a_{M}$, across the surface. The periodic nature of the coefficient $a\left(r_{\|}\right)$allows us to expect that functions satisfying Eq. (1) have, in general, the form of a Floquet function with $U\left(r_{\|} ; z\right)$ being a periodic part (factor) of this function. Therefore, we can assume that $U\left(r_{\|} ; z\right)$ is a function periodic on the surface and with $\boldsymbol{R}_{\|}$denoting the positions of the regions $I$ in the regular lattice (in Fig. 1 - a quadratic lattice) and with $\boldsymbol{G}$ denoting the vector of the lattice reciprocal to $\boldsymbol{R}_{\|}$- we can perform the following Fourier transforms leading us into the space of the reciprocal lattice $G$ :

$$
a\left(r_{\|}\right)=\sum_{\boldsymbol{G}} a(\boldsymbol{G}) \mathrm{e}^{\mathrm{i} \boldsymbol{G} \cdot r_{\|}},
$$




$$
U\left(\boldsymbol{r}_{\|} ; z\right)=\sum_{\boldsymbol{G}^{\prime}} U\left(\boldsymbol{G}^{\prime} ; z\right) \mathrm{e}^{\mathrm{i} \boldsymbol{G} \cdot \boldsymbol{r}_{\|} .}
$$

Insertion of $(3 a, b)$ into (1) leads to the following equation:

$$
\sum_{\boldsymbol{G}^{\prime}} \mathrm{e}^{\mathrm{i} \boldsymbol{G}^{\prime} \cdot r_{\|}}\left[\sum_{\boldsymbol{G}} a\left(\boldsymbol{G}^{\prime}-\boldsymbol{G}\right) U(\boldsymbol{G} ; z)+\frac{\partial U\left(\boldsymbol{G}^{\prime} ; \boldsymbol{z}\right)}{\partial z}\right]=0 .
$$

Since Eq. (4) has to be fulfilled for any $r_{\|}$, the expression in brackets has to vanish identically leading to

$$
\frac{\partial U(\boldsymbol{G} ; z)}{\partial z}+\sum_{\boldsymbol{G}^{\prime}} a\left(\boldsymbol{G}-\boldsymbol{G}^{\prime}\right) U\left(\boldsymbol{G}^{\prime} ; z\right)=0 .
$$

It is noteworthy that Eq. (5) has to be fulfilled for any $G$, so that we have arrived, in fact, at an infinite set of equations corresponding to an infinite number of vectors $\boldsymbol{G}$ (we shall also keep in mind that summation over $\boldsymbol{G}^{\prime}$ involves a vector $G$ as well).

\section{The notion of an effective surface parameter}

We shall restrict our further considerations to the case when the transform $U(G ; z)$ is factorizable with separation of the variables (this case comprises a wide class of physically meaningful problems):

$$
U(\boldsymbol{G} ; z)=u(\boldsymbol{G}) u(z)
$$

On insertion of (6) into Eq. (5) we get

$$
\sum_{\boldsymbol{G}^{\prime}}\left[a\left(\boldsymbol{G}-\boldsymbol{G}^{\prime}\right) u(z)\right] u\left(\boldsymbol{G}^{\prime}\right)+\frac{\partial u(z)}{\partial z} u(\boldsymbol{G})=0
$$

or, equivalently,

$$
\sum_{G^{\prime}} a\left(\boldsymbol{G}-\boldsymbol{G}^{\prime}\right) u\left(\boldsymbol{G}^{\prime}\right)+\left[\frac{1}{u(z)} \frac{\partial u(z)}{\partial z}\right] u(\boldsymbol{G})=0 .
$$

We now introduce the notion of an effective surface parameter, defined as follows:

$$
A=-\frac{1}{u(z)} \frac{\partial u(z)}{\partial z}
$$

so that $\mathrm{Eq}$. (7b) becomes

$$
\sum_{G^{\prime}} a\left(\boldsymbol{G}-\boldsymbol{G}^{\prime}\right) u\left(\boldsymbol{G}^{\prime}\right)-A u(\boldsymbol{G})=0 .
$$

Thus, the effective surface parameter can take only values that are eigenvalues of the preceding eigenproblem (9). Significantly, the set (9) does not involve the variable $z$, so that the spectrum of eigenvalues of the surface parameters is independent of the "position" of the surface and is completely determined by the composite structure of the surface expressed alone by the transforms $a\left(\boldsymbol{G}-\boldsymbol{G}^{\prime}\right)$.

The dimensionality (the number of equations) of the set (9) varies in accordance with our choice of the basis of vectors $\boldsymbol{G}$. Of course, strictly, (9) should be taken as an infinite set of equations and therefore those are the eigenvalues $A_{n}$ 
we obtain from the condition of the vanishing determinant of (9). However, for practical reasons, we shall be using a finite basis of values $G$; the number of the vectors forming the $\boldsymbol{G}$-basis is denoted by $N$. We can treat, at the same time, the number $N$ as the order of the approximation applied; thus, we can operate in the first, second, third and higher approximations $(N=1,2,3 \ldots)$. We establish the vectors $G$ as ordered according to their growing length, thus, with $G_{0}$ as zero vector we have the following notation:

$$
\boldsymbol{G}_{0}=0 ; \boldsymbol{G}_{1} ; \boldsymbol{G}_{2} ; \boldsymbol{G}_{3} ; \ldots \boldsymbol{G}_{N-1} .
$$

Let us first consider the lowest approximation $N=1$.

In a first approximation $(N=1)$ we take into consideration only one vector of the reciprocal lattice, namely $\boldsymbol{G}_{0}=0$. For $\boldsymbol{G}_{0}$, Eq. (9) simplifies considerably, namely it takes the following form:

$$
a(0) u\left(G_{0}\right)-A u\left(G_{0}\right)=0,
$$

which leads directly to but one "eigen"-value of the effective surface parameter

$$
A=a(0)
$$

and only a single characteristic equation (derived from (8)), which has to be fulfilled at the surface $z=z_{0}$ :

$$
\frac{\mathrm{d} u(z)}{\mathrm{d} z}+A u(z)=0 \quad \text { for } \quad z=z_{0} .
$$

Therefore, the lowest approximation (with $N=1$ ) gives one averaged value of the surface parameter, equal to the lowest, most strongly leading transform $a(0)$; in other words, in this approximation the composite nature of the surface has, as it were, decayed and been replaced by an averaged value of the surface parameter (homogeneous for the surface as a whole).

\section{Effective surface parameter eigenvalues}

The further steps bring us closer to the initial "mosaic" structure of the surface, since the expression for the eigenvalues of the surface parameter come to contain higher and higher Fourier components $a(\boldsymbol{G})$. In the next step of the approximation $(N=2)$ the $\boldsymbol{G}$-basis consists of two vectors, $\boldsymbol{G}_{0} \equiv 0$ and $\boldsymbol{G}_{1}$, and Eq. (9) leads to two equations respectively, namely

$$
\left.\begin{array}{c}
{[a(0)-A] u\left(G_{0}\right)+a\left(-G_{1}\right) u\left(G_{1}\right)=0,} \\
a\left(G_{1}\right) u\left(G_{0}\right)+[a(0)-A] u\left(G_{1}\right)=0 .
\end{array}\right\}
$$

The solvability condition for this set leads to two eigenvalues of the effective surface parameter

$$
A_{1 / 2}=a(0) \mp \sqrt{a\left(G_{1}\right) a\left(-G_{1}\right)} .
$$

Thus we obtain two characteristic equations of the type (12) on inserting therein respectively, $A_{1}$ and $A_{2}$ in place of $A$.

We readily extend our considerations to the case of arbitrary $N$ establishing the rule that the selection of a basis of $N$ vectors leads to $N$ eigenvalues of the effective surface parameter, to be denoted by

$$
A_{n} ; \quad n=1,2, \ldots N .
$$


One may say that the homogeneously-averaged value of the surface parameter obtained in the first approximation, undergoes now consecutive splitting into $2,3, \ldots N$ values, thus implying our consecutive approach to the fulfilment of the realistic (i.e. composite) surface condition. For each of the obtained eigenvalues $A_{n}$ the following boundary condition has to be fulfilled:

$$
\frac{\partial u(z)}{\partial z}+A_{n} u(z)=0, \quad n=1,2, \ldots N .
$$

This result indicates that our treatment of the composite surface conditions resides in transforming the initial composite surface condition (1) into an equivalent system of homogeneous surface conditions of the type (16) taking account of the multiple nature of the effective surface parameter expressed by its eigenvalues spectrum $A_{n}$. Thus, the rest of our work reduces to the standard analysis of the boundary problem with homogeneous surface, well known from the literature.

In the asymptotic approximation (for $N \rightarrow \infty$ ) the single effective parameter value (11) "attached" to the homogeneously averaged surface goes over into a band composed of an infinite number of sub-values (15). Thus, the higher the multiplicity $N$ considered, the closer we approach to the fulfilment of the realistic surface conditions. Practically, this amounts to the search for a sufficiently great $N$ ensuring good convergence of the results obtained.

\section{Outlooks}

The essence of the present paper resides in showing that the composite boundary condition (1), on subjection to the Fourier transformation, reduces to a specific eigenproblem (9) the eigenvalues of which constitute the spectrum of permitted values of an effective surface parameter, a novel quantity which we introduced to replace the composite surface parameter. In terms of effectivity, the procedure outlined above can be said to convert the composite surface into an equivalent "multiple homogeneous surface". A highly interesting aspect of this method resides in its future application to the study of boundary conditions in thin films and multilayers with periodically inhomogeneous surfaces or interfaces. These matters will be dealt with in separate papers.

\section{Acknowledgments}

The present work was supported by the grant No. 2 P03B 07916 from the Committee for Scientific Research.

\section{References}

[1] J.D. Joannopoulos, R.D. Meade, J.N. Winn, Photonic Crystals, Princeton University Press, New York 1995.

[2] Photonic Band Gap Materials, Ed. C.M. Soukoulis, Kluwer Academic, Dordrecht 1996.

[3] R. Hillebrand, W. Hergert, W. Harms, Phys. Status Solidi B 217, 981 (2000).

[4] M.S. Kushwaha, P. Halevi, G. Martinez, L. Dobrzynski, B. Djafari-Rouhani, Phys. Rev. B 49, 2313 (1994).

[5] J.O. Vasseur, B. Djafari-Rouhani, L. Dobrzynski, M.S. Kushwaha, P. Halevi, J. Phys., Condens. Matter 6, 8759 (1994). 
[6] J.O. Vasseur, B. Djafari-Rouhani, L. Dobrzynski, P.A. Deymier, J. Phys., Condens. Matter 9, 7327 (1997).

[7] Y. Tanaka, S. Tamura, Phys. Rev. B 58, 7958 (1998).

[8] Y. Tanaka, S. Tamura, Phys. Rev. B 60, 13294 (1999).

[9] J.O. Vasseur, L. Dobrzynski, B. Djafari-Rouhani, H. Puszkarski, Phys. Rev. B 54, 1043 (1996).

[10] M. Krawczyk, H. Puszkarski, Acta Phys. Pol. A 93, 805 (1998).

[11] M. Krawczyk, H. Puszkarski, Acta Phys. Superficierum 3, 89 (1999).

[12] A.A. Maradudin, A.R. McGurn, J. Opt. Soc. Am. B 10, 307 (1993).

[13] B. Djafari-Rouhani, A.A. Maradudin, J. Appl. Phys. 65, 4245 (1989).

[14] B. Djafari-Rouhani, A. Khelif, Vacuum 54, 309 (1999). 